\title{
Polite row about models in biology
}

\section{A public debate last week between Stuart Kauffman and John Maynard Smith entertained a London audience but left the status of modelling in biology ill-defined.}

DisAPPOINTINGLY little blood was spilled last week at the Linnean Society of London, where Professor John Maynard Smith (Sussex) and Dr Stuart Kauffman (Sante Fe Institute) had been tempted by the London Evolution Group to a public debate on Kauffman's mathematical models of evolution. In the event, they were restrained by their innate good manners, even their mutual liking for each other; Kauffman was one of Maynard Smith's first graduate students 30 years ago (and was probably as precocious then as he is still), and even claimed (physician as he is) to have cured Maynard Smith of pneumonia contracted on a visit to Chicago.

Last week's disappointment is real, because the advertised debate concerns a real issue. Kauffman's book The Origin of Order, in which his argument was rehearsed, caused something of a stir on its publication 18 months ago. A "mixed reception" is what the publishers (Oxford University Press) would have said (see Nature 365, 704; 1993). But last week, Kauffman would have known in advance that there were some friends among the audience. One distinguished British physicist volunteered that he had found the book to contain the only comprehensible account of the mathematics of evolution.

Kauffman's argument is straightforward enough. The principle of order is the self-organizing principle, right? And living things are self-organized entities; look how much like a cell a synthetic lysosome seems! The principles of neo-Darwinian evolution are heritable variation, the differential fitness of the inheriting individuals and then natural selection. So the central problem, to Kauffman, is to understand how self-organization and natural selection can operate simultaneously.

Nobody last week disagreed about the use of the likely number of viable offspring as a measure of fitness, but Kauffman sees the representation of the problem as what the mathematicians call a complex problem. There is a "fitness space" or "landscape" with as many dimensions as there are potentially heritable variations in which, as with complex problems in physics, there will be a very large number of local maxima, corresponding to phenotypically advantageous inherited configurations of variations. So how does natural selection, working on the variations of fitness a group of organisms has inherited, manage to beat a path to the global maximum of fitness?
Kauffman last week described a simple model of this state of affairs - a peptide with four amino acids for which the possible variations are the replacement of one amino acid by another. For what it is worth, the model is derived from one introduced by Maynard Smith some time ago (Nature 225, 563; 1970). To make the problem manageable, it is supposed that the only allowable amino acids in the four-peptide are alanine and glycine.

In principle, it should eventually be possible to compile a table of how all possible variations affect the effectiveness of the peptide in its biological role, and thus to map their fitness. Using arbitrarily chosen numbers, computer modelling shows the convergence of different peptides to a common end-result.

More generally, it is even now possible to demonstrate that there are indeed local maxima on the fitness landscape, and that the landscape as a whole is the more rugged as the fitness comes to depend the more extensively on variations at different sites in the four-peptide. The greater the interconnectedness, the more peaks appear in the landscape and the more difficult it is to reach local maxima, let along the global maximum, by random walking on the landscape.

So, says Kauffman, a smooth rather than a rough fitness landscape is a prerequisite for orderly evolution. How can that be achieved? Co-evolution is one way. ("If a frog evolves a sticky tongue to catch prey more effectively, the flies it catches had better evolve sticky feet.") But evolving organisms in general must change the ruggedness of the fitness landscape their successor organisms inherit.

There remain the grand questions the great Cambrian radiation of species, for example; was there something special about the fitness landscape of organisms then? Kauffman is a confessed devotee of the arguments (largely due to Per Bak) of the critical behaviour of self-organized systems, as in the formation of avalanches on the sides of a sandpile to which grains are added one at a time. The sandpile of self-organized living creatures must have reached a critical condition in the early Cambrian, spawning avalanches on its sides, each a new species or a genus.

Maynard Smith, an electrical engineer by background, a graduate student of J. B. S. Haldane and a modeller on his own account, is not one to deny mathematics a place in biology. Last week, he asked that "biologists should know a limitcycle when they see one" and know the causes of bifurcation in the evolution of chaotic systems. By way of illustration, he noted the apparent doubling of the number of segments in Drosophila (from seven to $7 \times 2=14$ ), suspecting that "Drosophila must have been reading my book".

Maynard Smith accepts the need for a relatively smooth fitness landscape, as defined, if evolution is to happen. But he could not follow Kauffman's supposition that self-organizing systems need external information to assemble, and suspected that Kauffman's view that evolving organisms influence their fitness landscape with a view to future evolution smacks of the Gaia hypothesis. The essence of his polite public dissent is simply put: the modelling of The Origin of Order notwithstanding, "We still do not know whether, if the environment were to stay constant, evolution would come to a stop".

Meanwhile, it is clear that Maynard Smith is not a devotee of Per Bak and his sandpiles. Among other things, he wondered what could be the correlate in the real world of the stream of sand-grains that eventually make the pile unstable. $\mathrm{He}$ also complained of an article in Nature (by this writer) "with punctuated equilibrium in the title" (Nature 371, 197; 1994). That concerns numerical simulations of evolutionary models in which the fitness of coevolving species is determined by random-number generators under particular rules. One conclusion is that it is possible to write the rules so that the initial state of the assemblage of species does not affect the outcome; another is that there can be circumstances when the system becomes unstable and several independent species emerge. Of this simulation and the sandpiles (as a model for real biology), Maynard Smith says: "I just find the whole enterprise contemptible".

What last week's audience made of the exchange is anybody's guess. Many beers later, the two protagonists did agree to try to define what their disagreement really is. After careful thought, Maynard Smith announced, "You see, Stu, I don't find it interesting". And that, of course, is what the argument should have been about. What is the place of modelling in biology? Can a model be heuristically valuable even when it entails only a sketchy correspondence with the real world? Maybe the Linnean Society should return to that charge.

John Maddox 